\title{
1 Biopsy quality is essential for preoperative prognostication in oral
}

tongue cancer

3 Ibrahim O. Bello", 2+*, Pia M. Wennerstrand ${ }^{2 *}$, Ilida Suleymanova ${ }^{3}$, Maria Siponen ${ }^{4,5}$, Ahmed

4 Qannam $^{1}$, Pentti Nieminen ${ }^{6}$, Ilmo Leivo ${ }^{7}$, Alhadi Almangush ${ }^{2,7,8, *}$, Tuula Salo ${ }^{2,3,9 *}$.

$6 \quad{ }^{1}$ Department of Oral Medicine and Diagnostic Sciences, King Saud University, Riyadh, Saudi Arabia.

$7 \quad{ }^{2}$ Department of Pathology, University of Helsinki, Helsinki, Finland.

$8{ }^{3}$ Department of Oral and Maxillofacial Diseases, Clinicum, Faculty of Medicine, University of Helsinki, Helsinki,

9 Finland.

$10{ }^{4}$ Institute of Dentistry, School of Medicine, Faculty of Health Sciences, University of Eastern Finland, Kuopio, Finland.

$11{ }^{5}$ Oral Health Teaching Clinic and Department of Oral and Maxillofacial Diseases, Kuopio University Hospital, Kuopio, 12 Finland.

$13{ }^{6}$ Medical Informatics and Data Analysis Research Group, University of Oulu, Oulu, Finland.

$14 \quad{ }^{7}$ Institute of Biomedicine, Pathology, University of Turku, Turku, Finland.

$15 \quad{ }^{8}$ Faculty of Dentistry, University of Misurata, Misurata, Libya.

$16{ }^{9}$ Cancer and Translational Medicine Research Unit, Medical Research Center Oulu, University of Oulu and Oulu 17 University Hospital, Oulu, Finland.

*Equal contribution

\section{${ }^{+}$Correspondence:}

Dr. Ibrahim O. Bello, BDS, PhD

23 Department of Oral Medicine and Diagnostic Sciences,

24 King Saud University, Riyadh, 11545, Saudi Arabia;

This article has been accepted for publication and undergone full peer review but has not been through the copyediting, typesetting, pagination and proofreading process, which may lead to differences between this version and the Version of Record. Please cite this article as doi: 10.1111/APM.13104

This article is protected by copyright. All rights reserved 
Department of Pathology, University of Helsinki,

Haartmaninkatu 3, Helsinki, FIN-00014, Finland.

27 Emails: ibello@ksu.edu.sa;

ibrahim.bello@helsinki.fi

Running title: Quality of biopsy and tongue cancer prognosis

31

\section{Summary}

Bello IO, Wennerstrand PM, Suleymanova I, Siponen M, Qannam A, Nieminen P, Leivo I, Almangush A, Salo T. Biopsy quality is essential for preoperative prognostication in oral tongue cancer

A role for incisional biopsy in preoperative prognostication is increasingly being advocated in oral tongue squamous cell carcinomas (OTSCC). Biopsies at two locations were compared and prognostic factors in biopsies and their corresponding resections were evaluated. A total of 138 OTSCC biopsy slides from Finland and Saudi-Arabia were compared for size (horizontal and vertical) and invasive front. The Finnish cases were assessed for tumor stroma ratio (TSR) and tumor-infiltrating lymphocytes (TILs) using light microscopy and digital image analysis assessment and compared. Furthermore, TSR, TILs, and previously analyzed budding and depth of invasion (BD) score in biopsies were compared with their evaluation in the corresponding resections. Fifty nine percent of Finnish and $42 \%$ of Saudi Arabian biopsies were $\geq 5 \mathrm{~mm}$ deep while $98 \%$ of Saudi-Arabian and $76 \%$ of Finnish biopsies were $\geq 5 \mathrm{~mm}$ wide. Assessment of invasion front was possible in $72 \%$ of Finnish in comparison to $40 \%$ of Saudi Arabian biopsies. There was $86.8 \%$ agreement between TSR and $75 \%$ agreement between TIL evaluation using light microscopy and digital assessment. Significant agreement was obtained on comparing the TSR $(\mathrm{P}=0.04)$ and $\mathrm{BD}(\mathrm{P}<0.001)$ values in biopsies and resections. Biopsies of $\geq 5 \mathrm{~mm}$ depth from representative OTSCC areas are essential for prognostic 
50 information. Clinical pathologists are advised to assess BD score and TSR for prognostic features in 51 such biopsies.

52

53

54 Keywords: oral tongue cancer; prognosis; quality of biopsy; preoperative

55

56

57

58

59

60

61

62

63

64

\section{Introduction}

66

67 Squamous cell carcinoma of the oral (mobile) tongue (OTSCC) contributes the highest number of 68 cases of oral squamous cell carcinoma (OSCC), and is associated with the lowest relative survival of

69 the different subsites within the oral cavity $(1,2)$. Incisional biopsy remains an indispensable 70 diagnostic tool for oral cancers. Cancer diagnosis is mandatorily followed by preoperative staging 71 (usually by clinical and imaging modalities) in order to determine the risk category and the most 72 appropriate treatment plan for the patient. The need for extending the role of the biopsy from being

This article is protected by copyright. All rights reserved 
only diagnostic to also include prognostication has recently been highlighted by some investigators (3, 4). In OSCC (and OTSCC), tumor grade of differentiation has not been found to be particularly useful in prognostication despite being the only universal routinely assessed histological feature in preoperative biopsies by pathologists (5-7).

In resection samples, several tumor-related histological features such as depth of invasion (DOI), worst pattern of invasion (WPOI), tumor budding, budding and depth of invasion (BD) score, as well as some stromal features such as tumor stroma ratio (TSR) and tumor-infiltrating lymphocytes (TILs) have been identified in several studies as being of significant prognostic potential in OTSCC (8-11). Notably, the latest AJCC/UICC TNM classification of head and neck tumors has significantly redefined the $\mathrm{T}$ staging, making DOI an integral part of the pathologic TNM classification with every $5 \mathrm{~mm}$ depth increment automatically upstaging the T stage to a higher one (T1 to T2 -5mm; T2 to T3 $10 \mathrm{~mm}$ ). Therefore, DOI is now as equally important as primary tumor horizontal dimension in the staging of T1-T3 tumors (12).

In order to minimize cases of under-treatment or unnecessary escalation in management, particularly regarding clinically negative neck (cN0) in early stage lesions, deeper biopsies are needed. They are necessary to explore as many histological prognostic details as possible before decisions are made regarding treatment. The assessment of several tumor and stromal prognostic features from an adequate biopsy coupled with adequate tumor imaging will result in a better characterized lesion and strongly enhance the probability that the best management method will be planned up-front (3).

This study sets its objective towards answering four questions: 1) Do OTSCC biopsies obtained from two different locations (Finland and Saudi Arabia) show similarities in width and depth? 2) In biopsies with optimal dimensions, can stromal features (namely TSR and TILs) be similarly assessed using either light microscopy or digital assessment (by machine learning with whole slide imaging, WSI) and how do the two methods agree with each other? 3) How do these stromal features, as well 
100 as our previously published budding (B) and depth of invasion (D) and (BD) scores from the same

101 biopsies compare with those obtained from the corresponding resection specimens?

102

103

104

105

106

107

108

109

110 Methods

111

112 The study was approved by the local institutional review boards of the 3 participating institutions:

113 Universities of Helsinki, Eastern Finland and King Saud, Saudi Arabia.

114 Histological slides of biopsies of OTSCC cases were randomly retrieved from the archives of two 115 Finnish University hospitals; Helsinki (HUH) and Kuopio (KUH), and King Saud University Oral 116 Histopathology Laboratory (KSU-OHL) in Saudi Arabia. Ninety-three cases were obtained from 117 Finland (HUH, 74 cases and KUH, 19 cases), while KSU-OHL contributed 45 cases, making 138 118 cases in total available for comparison. The inclusion criteria were the histological diagnosis of SCC 119 of the mobile tongue (irrespective of clinical staging), and the presence of tumor tissue in the patient 120 slides.

121 Biopsy dimensions measurement

This article is protected by copyright. All rights reserved 
122 The widest biopsy dimensions were measured in horizontal and vertical planes. A biopsy of at least 5

$123 \times 5 \mathrm{~mm}$ after tissue processing was considered optimal, including biopsies with tumor extending

124 beyond their margins (Figure 1).

125 Cancer tissue analyses

126 The DOI and carcinoma invasive front were evaluated, where possible, for all cases. DOI was defined 127 as a vertical line dropped from just underneath the epithelium (or its representation) to the end of the 128 most invasive part of the main tumor. Invasive front was taken at the advancing front (deepest point 129 of invasion) of the tumor in the connective tissue. Comparisons were made between the Finnish and 130 Saudi Arabian cases. In addition, previously published data of the BD score of the same biopsies and 131 corresponding resections by our group (for the Finnish cases) were retrieved and included (4).

\section{Stromal features}

133 For the Finnish cases, two stromal features, TSR and TILs were evaluated by light microscope, and 134 then digitally using WSI as described below (Figure 2). The technique for assessing TSR has been 135 previously well described $(10,13)$ : the area having the highest amount of stroma is selected with 136 tumor islands present at all borders under low magnification (x4), and thereafter, scored at higher 137 magnification (x10). The percentage of stroma relative to carcinoma tissue was then evaluated as the 138 TSR score. TILs were evaluated at the invasive front as also previously described: percentage of stroma occupied by lymphocytes. Scanning was done at low magnification (x4) and the TSR was assessed under high magnification (x20) (11). Based on previous studies, TSR and TILs were divided into high and low categories using $50 \%$ and $20 \%$ as the thresholds, respectively $(10,11)$. Light microscopic and digital assessment measurements were compared. The process was also done for resection slides using light microscopy only. TSR and TILs values from the biopsies were then 144 compared with the corresponding values from the resection sections. Only 42 resection histological slides were available corresponding to the 53 biopsies that were initially analyzed.

\section{Digital Assessment of Stromal features}

147 For digital computational assessment, the slides were scanned digitally (WSI) and analyzed using 148 QuPath (14). QuPath is an open source machine learning-based software for digital pathology image 
149 analysis. QuPath's ability to classify cell types within each tissue was applied to distinguish between

150 cancer cells and stromal TILs (Figure 2). The performance of a section image analysis was measured 151 by applying cell segmentation to entire tumor images using the cell detection algorithm in QuPath 152 with default settings. Tumor areas and stromal TILs were separated from the stroma by manually 153 drawing lines with the computer mouse on the images. Choice of assessment areas was made using 154 the same principles as used with light microscopy. Cellular detection settings were selected under 155 consultation with a pathologist and the evaluation carried out by a computer scientist (image analyst).

\section{Statistical analysis}

157 Frequency tables were made for comparison of biopsy quality between Finnish and Saudi Arabian 158 cases. Chi-square statistics or Fisher's exact test was used for evaluating statistical significance. 159 Additionally, mean age of the patients were compared using independent samples t-test. Cross160 tabulation of light microscopy versus digital assessment of TSR and TILs was performed. Agreement 161 between the measurements was evaluated using percentage agreement, Kappa and AC1 statistics (15) 162 Accuracy, sensitivity and specificity were calculated between stromal features in biopsies and 163 resections, and chi-square test was used for evaluation of significance. Analyses were done using IBM 164 SPSS version 23 and Matlab R 2016. 


\section{Demographic and biopsy characteristics}

175 The Saudi-Arabian patients were significantly younger than those of Finland with their mean age 176 about 7 years lower than the latter $(P=0.008,95 \%$ CI: 1.84-11.7). Two-thirds of Saudi-Arabian 177 patients were male while no sex predilection was observed in Finnish patients. (Table 1). No 178 significant difference was observed regarding the WHO histological grading between the two 179 countries.

180

181 182

183

184

185

186

187

188

189

190

191

192

193

194

195

196

197

198

199

200

\section{Biopsy dimensions and invasive front}

The vertical dimension of the biopsies was adequate $(\geq 5 \mathrm{~mm})$ in 74 cases $(54 \%)$ and $<5 \mathrm{~mm}$ in 64 cases (46\%) (Table 1, Figure 1). Fifty nine percent of biopsies from Finland and 42\% of those from KSA were $\geq 5 \mathrm{~mm}$. Most cases (84\%) from both centers were "optimal" in horizontal dimension. Clinicians from Saudi-Arabia appeared to take wider biopsies ( $\geq 5 \mathrm{~mm})$ than those from Finland (98\% KSA vs $76 \%$ Finnish). Regarding the invasive front, it was microscopically demonstrable in $62 \%$ of all the biopsy samples, and was more clearly identified Finnish biopsies than those from Saudi-Arabia (72\% Finnish vs 40\% KSA). The relationship of the invasive front and the DOI is shown in Figure 3. The optimality of a biopsy did not automatically translate to having assessable DOI. Some biopsies with depth $<5 \mathrm{~mm}$ had superficially invasive cancer from which the invasive front and stromal features could be adequately analyzed. Many others with vertical length $>5 \mathrm{~mm}$ did not have DOI or invasive front that could be assessed owing to tumor tissue extending beyond biopsy margins (Figure 3).

\section{Stromal features}

Of the 93 tumors assessed for TSR and TILs, only 53 biopsies were suitable for assessment by light microscopy. One of these cases could not be analyzed digitally making only 52 cases available for the latter (Figure 2). Agreement between light microscopy and digital measurements were compared using Cohen's kappa coefficient and was substantial for TSR (kappa value, 0.70) than TIL (kappa value, 0.52). The AC1 values were better or comparable to kappa's. The strength of the percentage 
201 agreement (87\%) and (75\%) respectively illustrate substantial agreement between both methods

202 (Table 2). A notable issue with digital analysis is the inclusion of unwanted cells (e.g. degenerated 203 muscle cells as tumor cells and lymphocytes as tumor cells or some tumor cell nuclei as lymphocytes) 204 or exclusion of relevant cells (e.g. some lymphocytes in TILs evaluation (Figure 2).

205

206

207

208

209

210

211

212

213

214

215

216

217

218

219

220

221

222

223

224

225

226

\section{Comparison of stromal features in biopsies and resection specimens}

For the 42 cases that simultaneously had both their biopsies and their resection specimens available, good level of agreement was observed in the case of TSR in biopsies when compared to resections (71\% accuracy, $P=0.04)$. For TIL, there was low agreement between biopsies and resections $(64 \%$ accuracy, $P=0.116)$ (Table 3).

\section{Combination of carcinoma and stromal features in biopsies and resections}

Using the same biopsy and resection samples, we have previously shown that BD score (which combines the depth of invasion and the budding of the cancer cells) has a good agreement between adequate biopsies and their resections $(83 \%, \mathrm{P}<0.001)(4)$. As shown in Table 4, this provides strong evidence for advising clinical pathologists to evaluate BD score (4) and TSR (present study) in biopsies.

\section{Discussion}

The primary role of OSCC biopsy is diagnostic but recently, there is increasing advocacy for a prognostic role for it $(3,4,16,17)$. Only optimal (at least $\geq 5 \mathrm{~mm}$ deep after tissue processing) biopsies can serve this role. Biopsies close to $10 \mathrm{~mm}$ in depth have been suggested to accurately predict the true DOI in at least $80 \%$ of OSCC patients, although surgeons consistently take biopsies $<5 \mathrm{~mm}$ in depth irrespective of their experience, tumor accessibility and size (3). Despite good accessibility, lack of obstructive structures (e.g. bone) and minimal risk of damage to important structures, the mean depth of OTSCC biopsies was still $<5 \mathrm{~mm}$ (3). Many biopsies in the present study were sufficiently wide but relatively shallow, especially those from KSA. DOI is now a clearly defined parameter as part of the pathologic T staging of OSCC by AJCC/UICC. Clinicians have even 
advocated estimating DOI in OSCC using bimanual palpation for clinical staging (18). Stromal features like TSR and TILs are better assessed in deeper biopsies where the invasive front including cancer cells growth pattern may be more clearly identified. A shallow biopsy may only be useful for the estimation of these features in superficially invasive tumors (Figure 3).

In this study, only $62 \%$ of biopsies had determinable invasive front. There was a significant contrast between the Finnish and Saudi-Arabian biopsies in relation to detecting the invasive front with far more of invasive front observed in the Finnish samples. Since the clinical staging of the KSA tumors was not available for this study, it is a plausible suggestion that the KSA tumors may comprise more advanced cases in which the invasive front is more difficult to obtain. Interestingly, it is noteworthy that most of the Finish tumors (73\%) were low-stage. The value of imaging methods in evaluating the DOI has been reported. However, it is possible to overestimate the DOI using imaging methods by as much as $3 \mathrm{~mm}$ when compared with anatomic pathological assessment $(19,20)$. A combination of clinical estimation and preoperative imaging evaluation of DOI, supplemented by further biopsy evaluation will greatly increase the accuracy of the tumor staging. Notably however, in some cases, it will still not be possible to measure DOI from biopsies due to problems like tissue fragmentation, distorted plane of sectioning, and samples with only epithelial components and no connective tissue interface. Even in resection samples, technical difficulties that may hamper the precise measuring of DOI have been highlighted in some studies $(21,22)$. Dhanda and colleagues (3) have suggested that standardization of all oral cancer biopsies using the punch technique will more likely increase the accuracy of the assessment of stromal to epithelial ratio, in addition to being a more proficient technique than the scalpel biopsy. All the biopsies assessed here were taken by scalpel blades, mostly in a wedge form with the surface forming the base of a triangle or the larger end of a trapezium. Using this method often leaves a very small area for interface analysis if the biopsy is taken from the central parts of the tumor. To reduce this problem, the general advice is to take the biopsy at the periphery of the lesion, especially in verrucopapillary lesions, alongside an adjacent "normal" tissue. However, taking more than one specimen may seem preferable, including the most severe (avoiding necrosis), internal regions with the deepest cancer growth $(23,24)$. In practice, since biopsies shrink after 
fixation, it is advisable to make the depth of the biopsy slightly more than the required $5 \mathrm{~mm}$. Some of these recommendations are illustrated in Figure 4.

The possibility of assessment of stromal histological prognostic features of OTSCC (e.g. TSR, TILs) offers an intriguing prospect in its preoperative staging and management planning (3). The prognostic potential of these features in OTSCC has been well-documented $(10,11)$. In this study, $43 \%$ of cases included in the evaluation of stromal features were found not to be suitable for such an assessment. A lot more cases would have been found suitable if a biopsy technique that could reliably sample both epithelial and stromal tissue is instituted (3). Many clinicians still rely on scalpel biopsies, and it may be sufficient that the only procedural modification needed is to make deeper incisions (24).

An important finding in this study is that not only is it possible that digital analysis can be used for assessing the stromal features in biopsies but that its agreement with light microscopic assessment is quite significant. In the biopsies, percentage agreement for the stromal features was between $75 \%$ and $87 \%$. All the digital measurements for this study were made by an image analyst with no prior pathology training. A pathologist was only on hand to guide on where measurements should be made. It could be argued that if a pathologist proficient in the use of digital measurement actually did the measurement, the agreement rate could be much higher. Many histopathology laboratories now have access to digital pathology which could be ultimately integrated into histopathology workflow and not only limited to research, teaching and external quality assurance practice (25). Ultimately using Qupath for stromal assessment could improve the preoperative tumor staging and risk assessment stratification to ensure that the most appropriate treatment is rendered, and obviate the need for several unplanned interventions thereafter.

The problem of lack of complete concordance between digital diagnosis and light microscopy has been highlighted in some recent systematic reviews of the subject $(26,27)$. Problems identified with digital microscopic diagnosis include limited image resolution, difficulties in identifying cell types and cellular structures, poor biopsy quality, lack of immunohistochemistry and special stains, poor 
282

283

284

285

286

287

288

289

290

291

292

293

294

295

296

297

298

299

300

301

302

303

304

305

306

307

308

309

310

assessment of nuclear atypia, grading of dysplasia and malignancy and poor identification of small objects $(26,27)$. In essence, digital microscopy has yet to reach the level presently attainable with light microscopy. Interestingly, in OSCC resections, a recent study proposed a relatively objective digital evaluation of TILs abundance (TILAb) in WSI by segmentation into tissue types (e.g. tumor and lymphocytes), and then using a deep convolutional neural network and binary classifier of tumorlymphocyte co-localization to estimate TILAb. TILAb was found to be a strong prognostic indicator of disease-free survival and better than manually assessed TILAb (28). Digital tumor staging and risk assessment is a more advanced procedure (despite the issues raised above being carried over into it) than digital diagnostics using WSI. Digital measurements as used in this work therefore needs to be further refined to eliminate unwanted cells and include relevant cells that it has excluded. Additionally, immunohistochemical staining as an adjunct may be very helpful in making QuPath to more efficiently identify tumor cells and lymphocytes when assessing TSR and TILs. It is a limitation of this study that the issues currently raised with digital microscopy could not be sufficiently addressed and this may have contributed to the discordance noted in light microscopic and digital measurements.

Regarding the comparison between biopsies and resections, only TSR showed significant agreement when the two were compared. The explanation is that TSR measurement is based on finding a single spot on the tumor slide for measurements to be taken. Conversely, TILs measurement depends on scanning the whole of the slide's tumor invasive front to arrive at the aggregated score. Pathologists are generally familiar with variations in lymphocytic host response in different parts of a resection. TILs in biopsies may therefore not be truly representative of the real picture. It provides only a snapshot of the TILs population in the area of the tumor from which the biopsy was taken. Similarly, due to lack of uniformity all around the tumor, discordant evaluation of the WHO histological grade in biopsies and resections has been previously reported (5). In general, if TILs score is high in a biopsy, it is an indication that it will be high in the resection while low value in a biopsy does not always imply low value in the resection. TSR appears not to be affected by the issues noted with TILs and therefore could possibly be added to the previously reported tumor histological factors of prognostic importance that can be evaluated in biopsies (Table 4).

This article is protected by copyright. All rights reserved 
312 In conclusion, this study showed that stromal prognostic features can be evaluated in optimal and 313 representative OTSCC biopsies, using both light microscopy and digital measurements. There is 314 relatively good agreement using both methods despite the small sample size available for this pilot 315 study, and also when compared with corresponding resection specimen. Digital evaluation needs 316 further refining and familiarity to oral and maxillofacial pathologists for easy and relatively accurate 317 evaluation of stromal features. Finally, we suggest that clinicians should take representative deep 318 biopsies ( $>5 \mathrm{~mm}$ ), and clinical pathologists should evaluate BD and TSR scores from those biopsies. 319 While considerations for complications such as bleeding and functional deficits may be of important 320 concern in biopsies and excision in other oral cavity subsites, it is often less pronounced in the oral 321 tongue (24). Clinicians should make the preparations needed to successfully obtain an adequate 322 biopsy while at the same time ensure that morbidity is pre-empted, especially in older patients (often 323 with co-morbid conditions) who constitute the bulk of patients with OTSCC.

324 Conflict of Interest: None declared by the authors

325 Ethical Consideration: The study was approved by the local institutional review boards of 326 Universities of Helsinki, Eastern Finland and King Saud, Saudi Arabia.

327 Informed Consent: Not applicable to this study and non-identifiable patient material used.

328 Word Count: 3090

329 AUTHORS' CONTRIBUTIONS

330 Study concept and design: TS, IOB, AA; Data acquisition and quality control: AA, IOB, PMW, AQ, 331 IL, MS, TS; Data analysis and interpretation: AA, IOB, PMW, IS; Statistical analysis: PN, IS; 332 Manuscript preparation: IOB, AA, PMW, TS; Manuscript editing and review: ALL AUTHORS 333 


\section{References}

337 1. $\mathrm{Ng} \mathrm{JH}$, Iyer NG, Tan $\mathrm{MH}$, Edgren G. Changing epidemiology of oral squamous cell carcinoma of the 338 tongue: A global study. Head Neck. 2017;39(2):297-304.

2. Listl S, Jansen L, Stenzinger A, Freier K, Emrich K, Holleczek B, et al. Survival of patients with oral cavity cancer in Germany. PLoS One. 2013;8(1):e53415.

3. Dhanda J, Uppal N, Chowlia H, Opie N, Al-Qamachi L, Shelat D, et al. Features and prognostic utility of 344 biopsy in oral squamous cell carcinoma. Head Neck. 2016;38 Suppl 1:E1857-62.

4. Almangush A, Leivo I, Siponen M, Sundquist E, Mroueh R, Makitie AA, et al. Evaluation of the budding and depth of invasion (BD) model in oral tongue cancer biopsies. Virchows Arch. 2018;472(2):231-6.

5. Dik EA, Ipenburg NA, Kessler PA, van Es RJJ, Willems SM. The value of histological grading of biopsy and resection specimens in early stage oral squamous cell carcinomas. J Craniomaxillofac Surg. 2018;46(6):1001-6.

6. Weijers M, Snow GB, Bezemer PD, van der Waal I. Malignancy grading is no better than conventional histopathological grading in small squamous cell carcinoma of tongue and floor of mouth: retrospective study in 128 patients. J Oral Pathol Med. 2009;38(4):343-7.

357 7. Keski-Santti H, Atula T, Tikka J, Hollmen J, Makitie AA, Leivo I. Predictive value of histopathologic parameters in early squamous cell carcinoma of oral tongue. Oral Oncol. 2007;43(10):1007-13.

8. Almangush A, Bello IO, Coletta RD, Makitie AA, Makinen LK, Kauppila JH, et al. For early-stage oral 361 tongue cancer, depth of invasion and worst pattern of invasion are the strongest pathological predictors for locoregional recurrence and mortality. Virchows Arch. 2015;467(1):39-46. 
364 9. Tam S, Amit M, Zafereo M, Bell D, Weber RS. Depth of invasion as a predictor of nodal disease and 365 survival in patients with oral tongue squamous cell carcinoma. Head Neck. 2019;41(1):177-84.

367 10. Almangush A, Heikkinen I, Bakhti N, Makinen LK, Kauppila JH, Pukkila M, et al. Prognostic impact of 368 tumour-stroma ratio in early-stage oral tongue cancers. Histopathology. 2018;72(7):1128-35.

11. Heikkinen I, Bello IO, Wahab A, Hagstrom J, Haglund C, Coletta RD, et al. Assessment of Tumorinfiltrating Lymphocytes Predicts the Behavior of Early-stage Oral Tongue Cancer. Am J Surg Pathol. 2019.

12. Brierley J, D., Gospodarowicz M, K., Wittekind C, editors. TNM Classification of Malignant Tumours.

374 8th ed. UK: John Wiley \& Sons, Ltd; 2017.

13. van Pelt GW, Kjær-Frifeldt S, van Krieken J, Al Dieri R, Morreau H, Tollenaar R, et al. Scoring the tumor-stroma ratio in colon cancer: procedure and recommendations. Virchows Arch. 2018;473(4):405-12. 14. Bankhead P, Loughrey MB, Fernandez JA, Dombrowski Y, McArt DG, Dunne PD, et al. QuPath: Open source software for digital pathology image analysis. Sci Rep. 2017;7(1):16878.

15. Gwet KL. Computing inter-rater reliability and its variance in the presence of high agreement. The British journal of mathematical and statistical psychology. 2008;61(Pt 1):29-48.

16. Seki M, Sano T, Yokoo S, Oyama T. Histologic assessment of tumor budding in preoperative biopsies to predict nodal metastasis in squamous cell carcinoma of the tongue and floor of the mouth. Head Neck. 2016;38 Suppl 1:E1582-90.

17. Jesinghaus M, Steiger K, Stögbauer F, Haller B, Kolk A, Straßen U, et al. Pre-operative cellular dissociation grading in biopsies is highly predictive of post-operative tumour stage and patient outcome in head and neck squamous cell carcinoma. Br J Cancer. 2020;122(6):835-46. oncology. 2018;9(1):116-20. 
395 19. Murakami R, Shiraishi S, Yoshida R, Sakata J, Yamana K, Hirosue A, et al. Reliability of MRI-Derived 396 Depth of Invasion of Oral Tongue Cancer. Acad Radiol. 2019;26(7):e180-e6.

20. lida Y, Kamijo T, Kusafuka K, Omae K, Nishiya Y, Hamaguchi N, et al. Depth of invasion in superficial oral tongue carcinoma quantified using intraoral ultrasonography. Laryngoscope. 2018;128(12):2778-82.

401 21. Berdugo J, Thompson LDR, Purgina B, Sturgis CD, Tuluc M, Seethala R, et al. Measuring Depth of 402 Invasion in Early Squamous Cell Carcinoma of the Oral Tongue: Positive Deep Margin, Extratumoral Perineural 403 Invasion, and Other Challenges. Head Neck Pathol. 2019;13(2):154-61.

405 22. Kukreja P, Parekh D, Roy P. Practical Challenges in Measurement of Depth of Invasion in Oral 406 Squamous Cell Carcinoma: Pictographical Documentation to Improve Consistency of Reporting per the AJCC 407 8th Edition Recommendations. Head Neck Pathol. 2020;14(2):419-27.

23. Lee JJ, Hung HC, Cheng SJ, Chiang CP, Liu BY, Yu CH, et al. Factors associated with underdiagnosis from incisional biopsy of oral leukoplakic lesions. Oral Surg Oral Med Oral Pathol Oral Radiol Endod.

$411 \quad 2007 ; 104(2): 217-25$.

412

24. Jeong WJ, Paik JH, Cho SW, Sung MW, Kim KH, Ahn SH. Excisional biopsy for management of lateral 414 tongue leukoplakia. J Oral Pathol Med. 2012;41(5):384-8. Arch. 2019;474(3):269-87. 
423 27. Azam AS, Miligy IM, Kimani PK, Maqbool H, Hewitt K, Rajpoot NM, et al. Diagnostic concordance and

424 discordance in digital pathology: a systematic review and meta-analysis. Journal of clinical pathology. 2020.

425

426 28. Shaban M, Khurram SA, Fraz MM, Alsubaie N, Masood I, Mushtaq S, et al. A Novel Digital Score for

427 Abundance of Tumour Infiltrating Lymphocytes Predicts Disease Free Survival in Oral Squamous Cell

428 Carcinoma. Sci Rep. 2019;9(1):13341.

429

430

431

432

433

434

435 Table 1. Demographic and biopsy characteristics of patients with OTSCC in Finland and Saudi

436 Arabia

$\begin{array}{llll}\text { HUH/KUH } & \text { KSU-OHL } & & \\ \text { (Finland ) } & \text { (Saudi Arabia) } & \text { Total } & \text { P-value }^{b} \\ \text { n=93 } & \text { n=45 } & & \end{array}$

Age

(mean; range) years ${ }^{\mathrm{a}}$

$61.8(27-93)$

$55.0(8-82)$

$59.5(8-93)$

0.008

Gender*

0.07

Male

$43(46 \%)$

$30(67 \%)$

73 (53\%)

Female

$43(46 \%)$

$15(33 \%)$

$58(42 \%)$

Missing

$7(8 \%)$

$7(5 \%)$ 


\begin{tabular}{llll}
\hline Superficial $(<5 \mathrm{~mm})$ & $38(41 \%)$ & $26(58 \%)$ & $64(46 \%)$ \\
Deep $(\geq 5 \mathrm{~mm})$ & $55(59 \%)$ & $19(42 \%)$ & $74(54 \%)$
\end{tabular}

\section{Horizontal Dimension}

$0.001 *$

Small $(<5 \mathrm{~mm})$

$22(24 \%) \quad 1(2 \%)$

$23(17 \%)$

Wide ( $\geq 5 \mathrm{~mm})$

$71(76 \%) \quad 44(98 \%)$

$115(83 \%)$

\section{Invasive front}

Absent

$26(28 \%)$

$27(60 \%)$

0.001

Present

$67(72 \%)$

$18(40 \%)$

$0.06^{*}$

\section{Tumor Grade}

Well (1)

$41(44 \%)$

$29(64 \%)$

$70(51 \%)$

Moderately (2)

$36(39 \%)$

$13(29 \%)$

$49(35 \%)$

Poorly (3)

$16(17 \%)$

$3(7 \%)$

$19(14 \%)$

\section{Clinical stage $^{\mathrm{a}}$}

$\mathrm{I} / \mathrm{II}$

$\mathrm{III} / \mathrm{IV}$

Missing
$68(73 \%)$

$18(19 \%$

$7(8 \%)$
$45(100 \%)$

437

$438 \quad$ aAge and gender data were only available in 86 cases for HUH and KUH patients, while the 439

440 clinical staging was not available for KSU/OHL as only pathology reports were provided.

441

442

This article is protected by copyright. All rights reserved 
Table 2. Analysis of agreement between use of light microscopy and digital assessment in biopsies

\begin{tabular}{|c|c|c|c|c|c|c|c|c|c|c|}
\hline $\begin{array}{c}\text { BIOPSIES } \\
\mathrm{N}=53^{\mathrm{a}}\end{array}$ & $\begin{array}{l}\text { TSR Low } \\
\text { (VM) }\end{array}$ & $\begin{array}{l}\text { TSR High } \\
\text { (VM) }\end{array}$ & $\begin{array}{c}\text { Kappa } \\
\text { value }\end{array}$ & $\begin{array}{l}\text { AC1 } \\
\text { value }\end{array}$ & $\begin{array}{c}\text { Agreement } \\
\%\end{array}$ & $\begin{array}{l}\text { TIL Low } \\
\text { (VM) }\end{array}$ & $\begin{array}{l}\text { TIL High } \\
\text { (VM) }\end{array}$ & $\begin{array}{c}\text { Kappa } \\
\text { Value }\end{array}$ & $\begin{array}{l}\text { AC1 } \\
\text { value }\end{array}$ & $\begin{array}{c}\text { Agreement } \\
\%\end{array}$ \\
\hline & & & 0.695 & 0.769 & $86.8 \%$ & & & & & \\
\hline TSR Low (LM) & 33 & 6 & & & & & & & & \\
\hline TSR High (LM) & 1 & 13 & & & & & & & & \\
\hline & & & & & & & & 0.520 & 0.502 & $75.0 \%$ \\
\hline TIL Low (LM) & & & & & & 21 & 12 & & & \\
\hline TIL High (LM) & & & & & & 1 & 18 & & & \\
\hline
\end{tabular}

${ }^{\mathrm{a}}$ One case of TIL was not determinable by virtual microscopy resulting in 52 cases

$\mathrm{LM}=$ light microscopy; $\mathrm{VM}=$ virtual microscopy (digital assessment) 
Table 3. Predictive analysis of agreement between biopsy samples (using light microscopy) and resection samples (light microscopy) in the same patients $(n=42)$

\begin{tabular}{|c|c|c|c|c|c|c|c|c|c|c|c|c|}
\hline \multirow[b]{2}{*}{ Biopsies } & \multicolumn{6}{|c|}{ Resection (LM) } & \multicolumn{6}{|c|}{ Resection (LM) } \\
\hline & $\begin{array}{l}\text { TSR } \\
\text { Low }\end{array}$ & $\begin{array}{l}\text { TSR } \\
\text { High }\end{array}$ & $\begin{array}{c}\text { Accuracy } \\
\%\end{array}$ & $\begin{array}{c}\text { Sensitivity } \\
\%(\mathrm{CI})\end{array}$ & $\begin{array}{c}\text { Specificity } \\
\%(\mathrm{CI})\end{array}$ & P-value & $\begin{array}{l}\text { TIL } \\
\text { Low }\end{array}$ & $\begin{array}{r}\text { TIL } \\
\text { High }\end{array}$ & $\begin{array}{c}\text { Accuracy } \\
\%\end{array}$ & $\begin{array}{c}\text { Sensitivity } \\
\%(\mathrm{CI})\end{array}$ & $\begin{array}{c}\text { Specificity } \\
\%(\mathrm{CI})\end{array}$ & P-value ${ }^{\mathrm{a}}$ \\
\hline & & & 71.4 & 85.7 & 42.9 & 0.040 & & & & & & \\
\hline TSR Low (LM) & 24 & 8 & & $(67.3-96.0)$ & $(17.7-71.1)$ & & & & & & & \\
\hline TSR High (LM) & 4 & 6 & & & & & & & & & & \\
\hline & & & & & & & & & 64.3 & 75.0 & 50.0 & 0.116 \\
\hline TIL Low (LM) & & & & & & & 18 & 9 & & $(53.3-90.2)$ & $(26.0-74.0)$ & \\
\hline TIL High (LM) & & & & & & & 6 & 9 & & & & \\
\hline
\end{tabular}

a $\mathrm{P}$-value of chi-square test; $\mathrm{CI}=$ Confidence interval; $\mathrm{LM}=$ light microscopy; $\mathrm{TSR}=$ tumor stroma ratio; $\mathrm{TIL}=$ tumor infiltrating lymphocyte 
Table 4. Predictive value of histopathological prognostic markers in biopsy specimen

\begin{tabular}{|c|c|c|c|c|c|}
\hline Parameters & $\begin{array}{l}\text { Pre-operative vs post- } \\
\text { operative matching }\end{array}$ & $\begin{array}{l}\text { Sensitivity } \\
(95 \% \mathrm{CI})^{\mathrm{a}}\end{array}$ & $\begin{array}{l}\text { Specificity } \\
(95 \% \mathrm{CI})^{\text {a }}\end{array}$ & P-value & Data source \\
\hline Depth of invasion & $77 \%$ & $77.1 \%(65.6-86.3 \%)$ & $76.7 \%(57.2-90.1 \%)$ & $<0.001$ & {$[4]$} \\
\hline Budding & $82 \%$ & $59.1 \%(43.3-73.7 \%)$ & $100 \%(96.3-100 \%)$ & $<0.001$ & {$[4]$} \\
\hline BD score & $83 \%$ & $57.1 \%(39.4-73.7 \%)$ & $96.9 \%(89.3-99.6 \%)$ & $<0.001$ & {$[4]$} \\
\hline Tumor stroma ratio & $73 \%$ & $85.7 \%(67.3-96.0 \%)$ & $42.9 \%(17.7-71.1 \%)$ & 0.04 & [This study] \\
\hline Tumor-infiltrating lymphocytes & $64 \%$ & $75 \%(53.3-90.2 \%)$ & $50 \%(26.0-74.0 \%)$ & 0.116 & [This study] \\
\hline
\end{tabular}

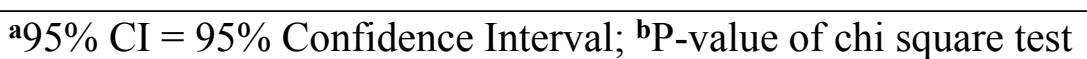



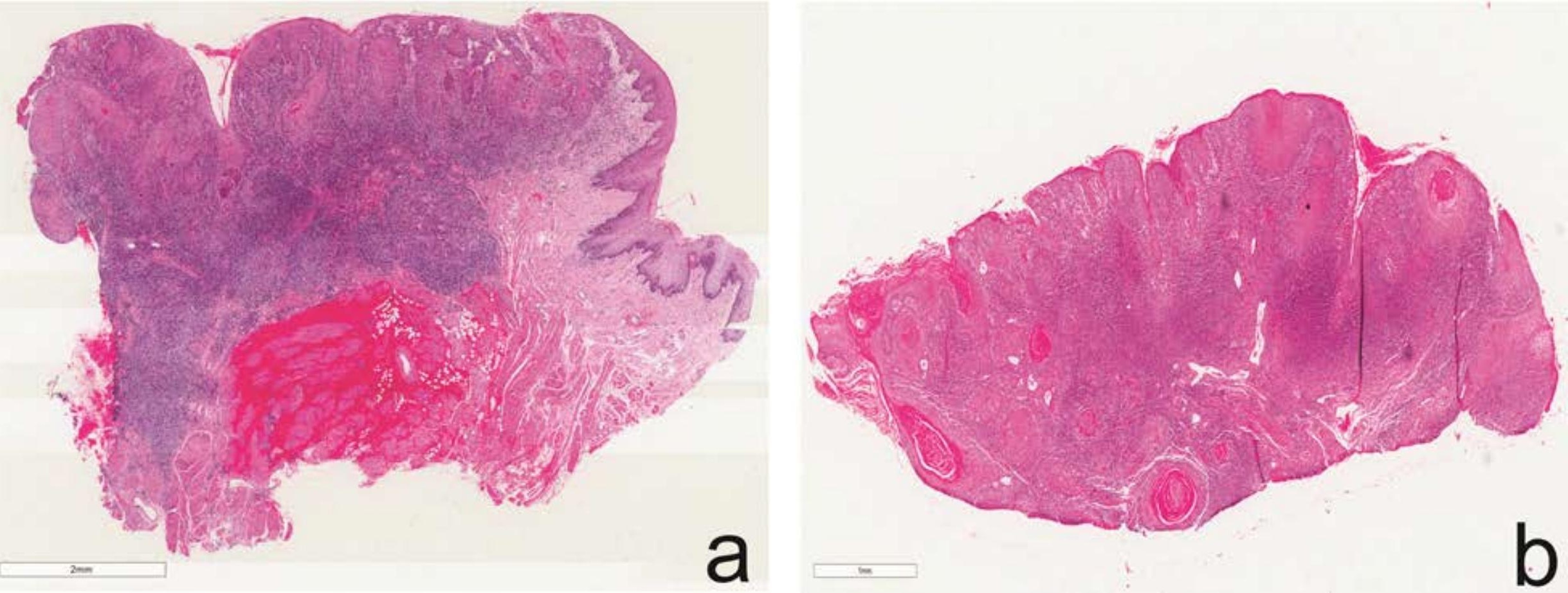


\section{8 biopsies}

Finland $(n=93)$

QA (biopsies)

\begin{tabular}{|l|c|c|c|}
\hline \multirow{2}{*}{$\begin{array}{l}\text { Vertical } \\
\text { depth }\end{array}$} & \multicolumn{3}{|c|}{ Invasive front } \\
\cline { 2 - 4 } & Yes & No & Total \\
\hline$<5 \mathrm{~mm}$ & 20 & 18 & 38 \\
\hline$\geq 5 \mathrm{~mm}$ & 47 & 8 & 55 \\
\hline
\end{tabular}

$\downarrow$

Biopsies TSR, TILs $(n=53)$

- LM

- $\mathrm{VM}$ *
Saudi Arabia $(n=45)$

QA (biopsies)

\begin{tabular}{|l|c|c|c|}
\hline \multirow{2}{*}{$\begin{array}{l}\text { Vertical } \\
\text { depth }\end{array}$} & \multicolumn{3}{|c|}{ Invasive front } \\
\cline { 2 - 4 } & Yes & No & Total \\
\hline$<5 \mathrm{~mm}$ & 7 & 19 & 26 \\
\hline$\geq 5 \mathrm{~mm}$ & 11 & 8 & 19 \\
\hline
\end{tabular}

Biopsies and corresponding

resections $(n=42)$

TILs, TSR (LM) 


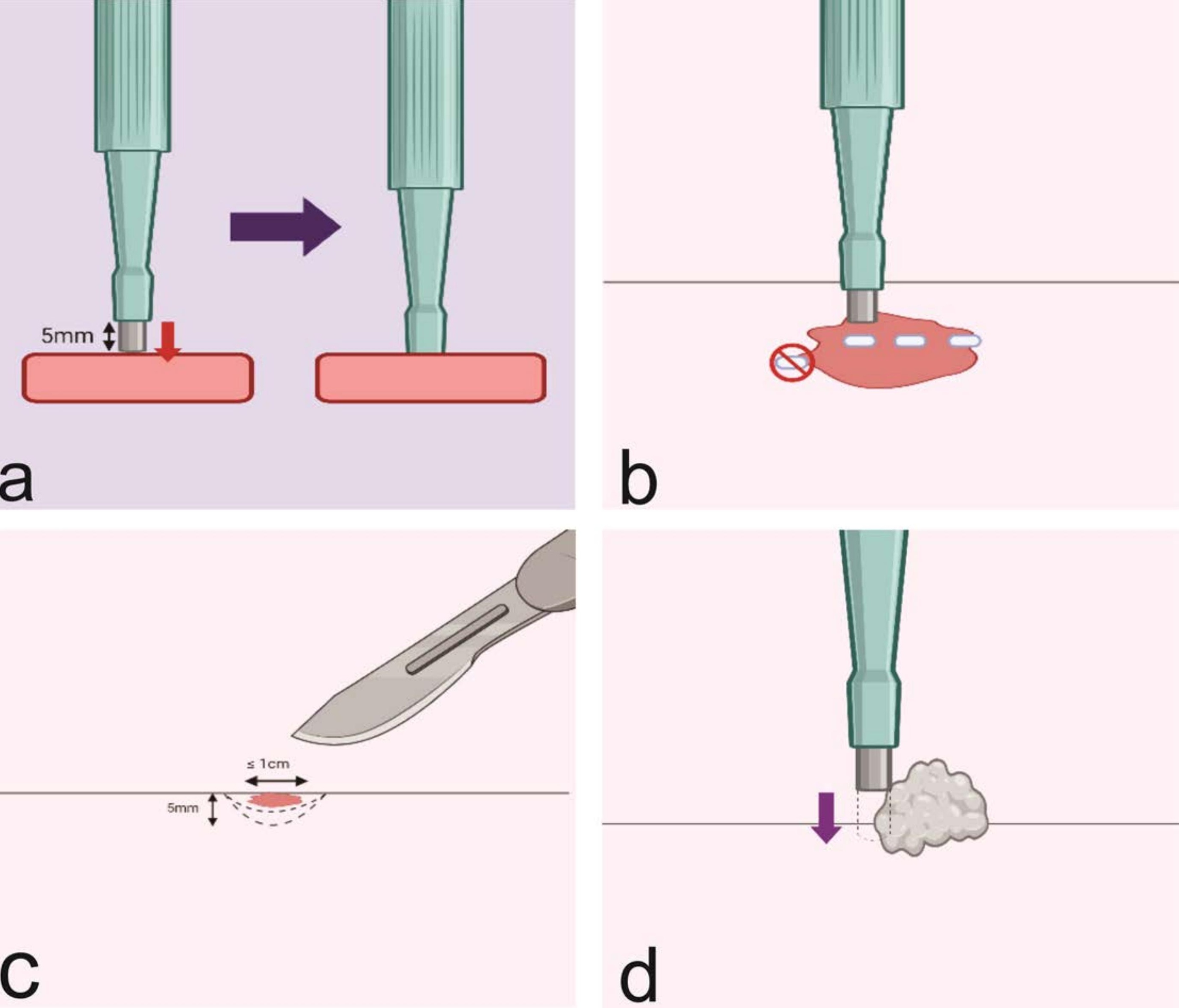

